Fetal and Pediatric Pathology, 00:1-5, 2014 Copyright@ Informa Healthcare USA, Inc. ISSN: $1551-3815$ print / 1551-3823 online
DOI: $10.3109 / 15513815.2014 .987934$ informa

\title{
Segmental Absence of Intestinal Musculature - Presentation in a 10-Year-Old Boy with an Extensive Involved Segment
}

\author{
Daniel Abensur Athanazio, ${ }^{1, *}$ Mateus T. Amaral Rocha, ${ }^{2}$ Angela \\ P. Mattos, ${ }^{3}$ Tereza Cristina Medrado Ribeiro, ${ }^{3}$ Ney Cristian Boa Sorte, ${ }^{3}$ \\ Luiz Antonio R. Freitas, ${ }^{1}$ and Luciana R. Silva ${ }^{1}$ \\ ${ }^{1}$ Federal University of Bahia, Salvador, Brazil,; ${ }^{2}$ Hospital Universitário Professor Edgard \\ Santos, Salvador, Brazil; ${ }^{3}$ Faculty of Medicine, Federal University of Bahia, Salvador, Brazil
}

\begin{abstract}
Segmental absence of the intestinal musculature is a rare cause of acute abdomen most likely associated with intestinal perforation and sepsis in neonates and adults. We present a case of a 10-year-old boy who developed acute abdomen and was treated with right hemicolectomy and partial resection of the ileum. The ileum showed a $20-\mathrm{cm}$-long stenotic segment showing prominent thinning of the intestinal wall. This case occurred in an older child, which appears to be rare when comparing the literature. The bowel defect was also the largest described to date, indicating the potential effect of an atonic segment leading to obstructive disease.
\end{abstract}

Keywords congenital abnormalities, peritonitis, ileum

\section{INTRODUCTION}

Segmental absence of the intestinal musculature (SAIM) is a rare clinicopathological entity characterized by thinning or complete absence of the muscularis propria with preservation of the mucosa[1]. Patients with SAIM usually present with progressive acute abdomen that is most likely associated with intestinal perforation and sepsis.[1, 2] Less commonly, patients may present with more prolonged clinical features such as intolerance to feeding and bilious vomiting[1].

The typical pathology of SAIM is of an isolated affected segment (usually less than $5 \mathrm{~cm}$ long) surrounded by normal bowel or bowel loops with preserved layers showing features of perforation and acute peritonitis. The diagnosis is dependent on histopathologic demonstration of absent muscularis propria[1, 2].

SAIM has been reported in isolated case reports and small series (approximately 20 cases to date), usually associated with prematurity; most cases share presentation within the first 99 days of life[1-4].

\section{CASE REPORT}

A 10-year-old boy sought medical assistance due to pain in the upper quadrants and postprandial vomiting. An upper GI endoscopy-based biopsy revealed mild atrophy

Received 14 April 2014; Revised xx xxxx; accepted 11 November 2014.

Address correspondence to Daniel Abensur Athanazio, Federal University of Bahia, Salvador, 


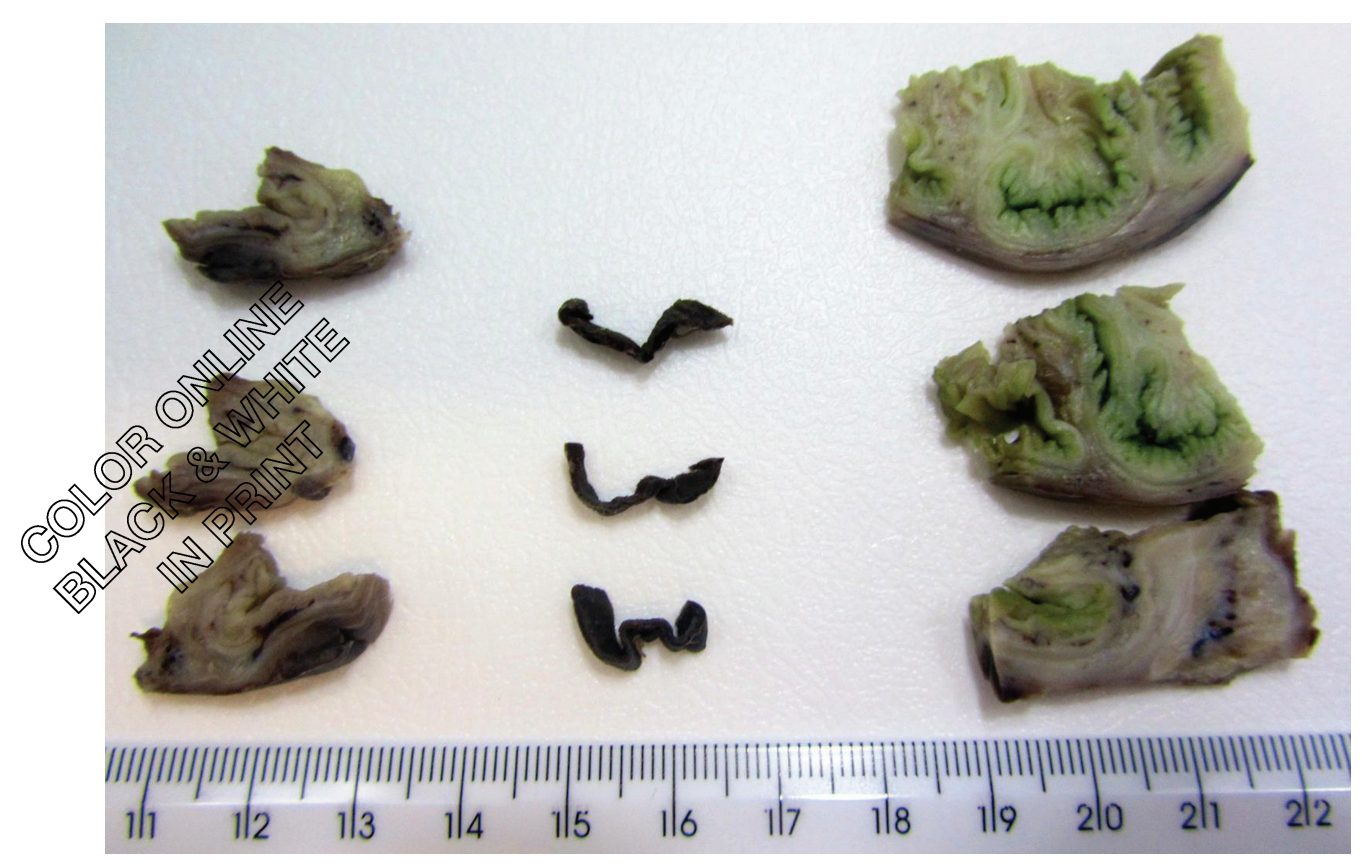

Figure 1. Gross appearance: the cut surface of the proximal 8-cm segment with no gross abnormalities (left); a 20-cm-long stenotic segment showing a reddish appearance on both serosal and mucosal surfaces and prominent thinning of the intestinal wall (center); and a distal 27-cm-long segment of ileal loops with preserved thickness of the intestinal wall but with prominent fibrous adherences between them (right).

(villous to crypt ratio of 3:1 to 2:1) and chronic inflammation at the duodenal mucosa. Detection of serum anti-gliadin IgA yielded weak reactivity. The patient was recommended to adhere to a gluten-free diet; however, this recommendation was never strictly followed. The patient's symptoms worsened during the following 45 days and were associated with the onset of fever. He developed signs of acute abdomen of probable obstructive origin and was treated with a right hemicolectomy and partial resec-

40 tion of the ileum. Examination of the surgical specimen (by the original pathology laboratory and on consultation of the specimen and slides) yielded the same findings. The right colon measured $18 \mathrm{~cm}$, and the appendix measured $9 \mathrm{~cm}$. The ileum measured $55 \mathrm{~cm}$ and could be grossly divided into three segments: an $8 \mathrm{~cm}$ proximal segment with no gross abnormalities; a 20 -cm-long stenotic segment showing a red-

45 dish appearance on both serosal and mucosal surfaces and prominent thinning of the intestinal wall; and a distal 27-cm-long segment of ileal loops with preserved thickness of the intestinal wall but with prominent fibrous adherences between them (Figure 1). The external surface of the right colon, appendix and ileum showed deposits of fibrinous/fibrous material suggestive of an organizing peritonitis. Microscopic ex50 amination of slides confirmed recent fibrosis and granulation tissue on the surface of the colon, appendix, and ileum. All three also showed marked reactive lymphoid aggregates. The most striking feature at histology was the absence of muscularis propria along the whole $20 \mathrm{~cm}$ ileal segment with grossly stenotic appearance associated with thinning of the intestinal wall (Figure 2). The submucosa showed remarkable conges55 tion. The surgical specimen also had five regional lymph nodes with reactive follicular and sinusal hyperplasia. 


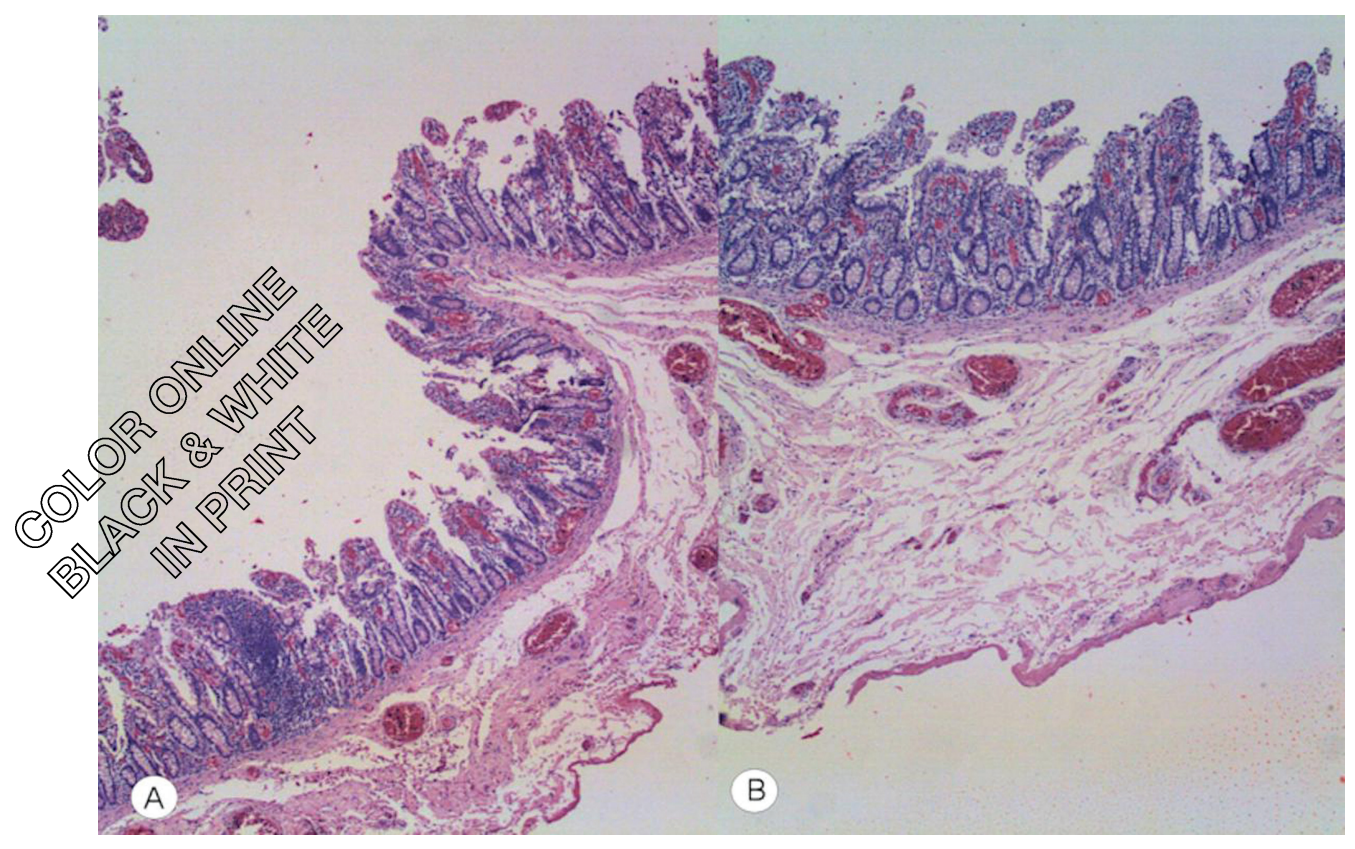

Figure 2. Absence of the muscular wall (A, $40 \times$ magnification and B, $100 \times$ magnification).

After surgery, the patient suffered intra-abdominal abscess and septic shock, which were treated with parenteral nutrition and antibiotics. After discharge, the family was recommended to seek a specialized service of pediatric gastroenterology.

At admission, the patient presented with severe malnutrition. He weighed $16.3 \mathrm{Kg}$ with a $Z$ score of -5.64 for height and of -2.45 for age. The patient had a history of birth by vaginal delivery at $2800 \mathrm{~g}$ (full term) without intercurrences. Tests for Giardia in feces and Mycobacterium spp. in gastric aspirate were negative. Chloride sweat was normal. Thyroid function was normal. Imaging tests (computed tomography and ultrasonography) 2 months after surgery showed diffuse thickening of small bowel loops. Ultrasonography showed also mild ascites. A new duodenal biopsy showed no signs of celiac disease. Five months after surgery, the patient still shows severe malnutrition and has developed recurrent episodes of respiratory tract infections.

\section{DISCUSSION}

Most reported cases of SAIM involve short affected segments. It has been stated that affected segments may be nearly normal at visual/gross inspection or may look like a small herniation of the intestinal wall. In such cases, the most obvious consequence is the risk of perforation $[1,3]$. In some of the literature, some speculate that SAIM may be a common condition that is not recognized. One argument favoring this hypothesis is the observation that spontaneous intestinal perforation, which is characterized by localized perforation without clinical and histologic features of necrotizing enterocolitis, is often associated with thinning muscularis propria[5].

In this present case, however, the total extent of the lesion was larger than in previous reports, and the age of presentation was considerably higher than expected. Rather than a herniation, SAIM presented as a stenotic segment, which most likely caused intestinal obstruction due to segmental atony of the ileum. 


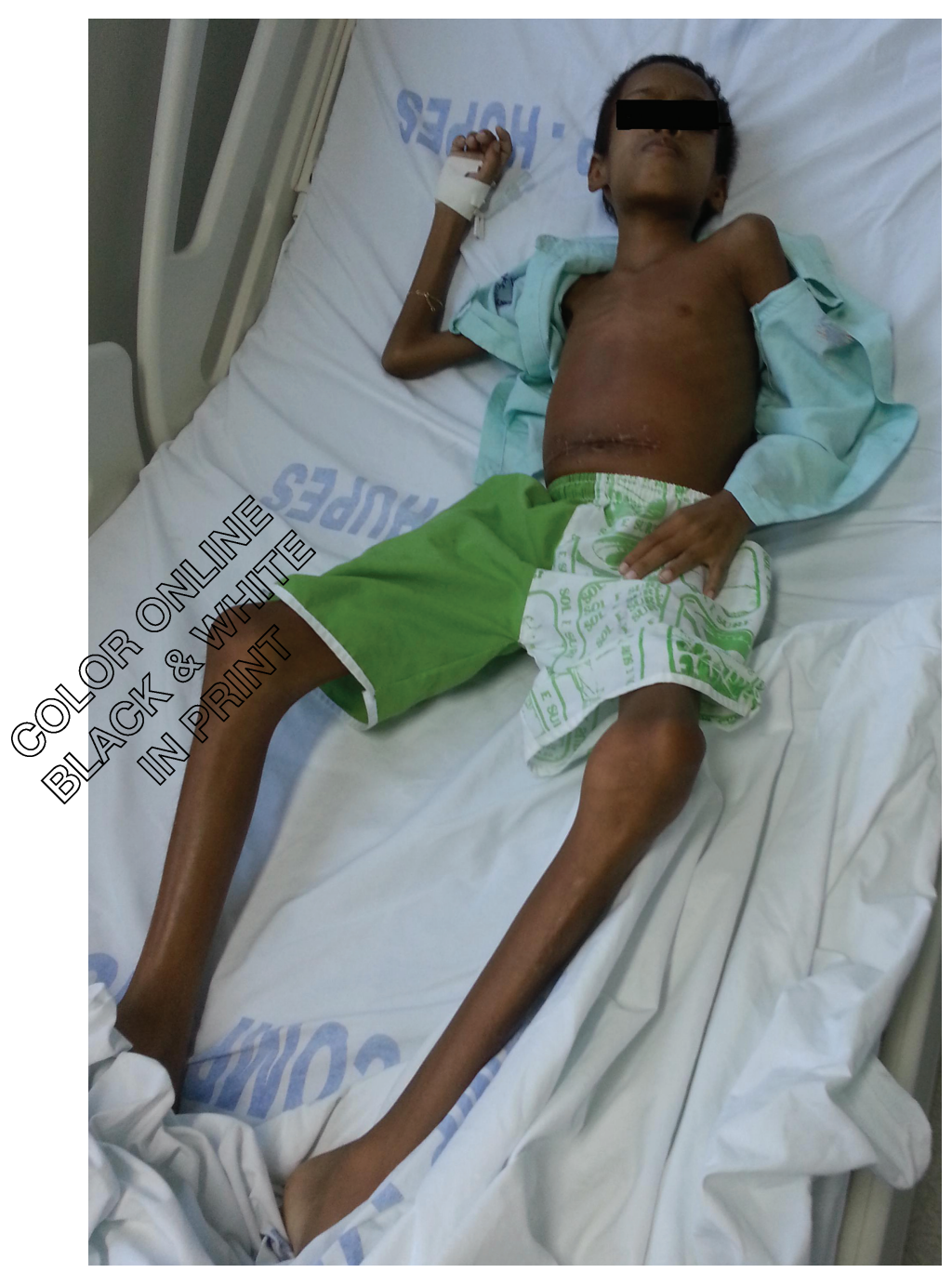

Figure 3. Five months after surgery, the patient shows severe malnutrition.

While well described in neonates, presentation of SAIM in older children and adults is exceptionally rare. To the best of our knowledge, there are three reports of SAIM in adults in the literature. Two reports were of a 34-year-old male and a 64-year-old female presenting as recurrent bowel obstruction and perendoscopic perforation, respectively[6, 7]. The former case is similar to the case reported here with regard to the extent of the SAIM segment (11 cm long in mid-jejunum) and the association with obstructive symptoms[6]. The third report was somewhat different in presentation, with multiple large diverticula in the jejunum as an incidental finding at surgery in a middle-aged male patient [8].

In conclusion, SAIM is a rare but well-described cause of bowel obstruction and perforation. It typically presents as small defects of the bowel wall in neonates and is usually associated with prematurity. It has also been reported in adults as an incidental finding or associated with perforation/obstruction. In the present case, an older child (10-year old) presented with severe malnutrition, a previous history of bowel obstruction and pathologic features of organizing peritonitis. The bowel defect was also the 
largest described to date, indicating the potential effects of an atonic segment leading to obstructive disease.

\section{Declaration of Interest}

The authors report no conflicts of interest. The authors alone are responsible for the 100 content and writing of the article.

\section{REFERENCES}

[1] Davis JS, Ryan ML, Shields JM, et al. Segmental absence of intestinal musculature: an increasingly reported pathology. J Pediatr Surg 2012;7:566-1571.

[2] Moore SW, Schneider JW, Kaschula RO. Unusual variations of gastrointestinal smooth muscle ab- 105 normalities associated with chronic intestinal pseudo-obstruction. Pediatric Surgery International 2002;8:3-20.

[3] McCarthy DW, Qualman S, Besner GE. Absent intestinal musculature: anatomic evidence of an embryonic origin of the lesion. J Pediatr Surg 1994;9:476-1478.

[4] Huang SF, Vacanti J, Kozakewich H. Segmental defect of the intestinal musculature of a newborn: 110 evidence of acquired pathogenesis. J Pediatr Surg 1996;1:21-725.

[5] Pumberger W, Mayr M, Kohlhauser C, Weninger M. Spontaneous localized intestinal perforation in very-low-birth-weight infants: a distinct clinical entity different from necrotizing enterocolitis. $\mathrm{J} \mathrm{Am}$ Coll Surg 2002;95:96-803.

[6] Tawfik O, Newell B, Lee KR. Segmental absence of intestinal musculature in an adult. Dig Dis Sci 115 1998;3:97-399.

[7] Darcha C, Orliaguet T, Levrel O, et al. [Segmental absence of colonic muscularis propria. Report of a case in an adult]. Annales de Pathologie 1997;7:1-33.

[8] Aldalati O, Phelan C, Ibrahim H. Segmental absence of intestinal musculature (SAIM): a case report in an adult. BMJ Case Rep 2009;009. 